\title{
Prime time for veno-arterial extracorporeal membrane oxygenation in 24-7 interventional cardiology center?
}

\author{
Marko Noc \\ Center for Intensive Internal Medicine, University Medical Center Ljubljana, Ljubljana, Slovenia
}

Postep Kardiol Inter 2015; 11, 4 (42): 285-287

DOI: $10.5114 /$ pwki.2015.55598

Because of continuous improvements in emergency prehospital services, high-volume ST-elevation myocardial infarction (STEMI) networks are nowadays admitting increasing numbers of patients with hemodynamic deterioration including profound cardiogenic shock and refractory cardiac arrest. The intra-aortic balloon pump (IABP) unfortunately offers only limited hemodynamic support without evidence of improved outcomes in the randomized SHOCK-IABP trial [1]. Accordingly, in a busy 24-7 catheterization laboratory, there is an unmet need for an active full-flow device which can be quickly and easily implanted by an interventional cardiologist. Such a device would promptly stabilize a patient, allow subsequent percutaneous coronary intervention $(\mathrm{PCl})$ in a more stable condition and buy time for myocardial recovery or serve as a bridge to a long-term assist device or heart transplantation. Various devices such as the Impella or TandemHeart, as well as veno-arterial extracorporeal membrane oxygenation (VA ECMO), are nowadays available. Each of these devices has advantages and disadvantages in terms of complexity, implantation procedure, hemodynamic characteristics, complications and costs. Importantly, none of the devices has yet been demonstrated to improve clinical outcomes, although available randomized trials are significantly underpowered.

In this issue of the journal, Litwiński et al. report a non-ST-elevation myocardial infarction (NSTEMI) patient with life-threatening iatrogenic left main dissection during coronary angiography which could not be solved by stenting [2]. As expected, the patient immediately developed cardiogenic shock refractory to mechanical ventilation and inotropes. No hemodynamic support was used before or during the transport to a tertiary center, where he underwent immediate coronary artery bypass graft surgery (CABG). Despite surgical revascularization, severe left ventricular pump failure refractory to pharma- cological treatment and IABP persisted. The patient was therefore put on postcardiotomy central VA ECMO for 14 days, survived to hospital discharge and is now a candidate for heart transplant.

What can we learn from this case report? Obviously, with such an unresolved complication and without active circulatory support, the patient was very fortunate to survive transportation to a tertiary institution. I suspect there was a partial restoration of anterograde flow despite dissection, which, together with presence of the right dominant coronary system, kept him from refractory cardiac arrest. The majority of such patients are not so lucky. This brings us to the question of whether it is necessary to have an effective hemodynamic support device in every 24-7 catheterization laboratory to bridge severe hemodynamic deterioration which may be present in some patients already at admission and persist despite successful $\mathrm{PCl}$, or develop, fortunately very rarely, as a consequence of complications or failed PCI.

In such critical situations, we would use VA ECMO, which is an active technique, able to generate normal cardiac output and thereby completely replace the heart and lung $[3,4]$. Because of technical developments, the VA ECMO console has become smaller and priming may nowadays be safely performed by trained nurses in 5 to 10 min in the catheterization laboratory (Figure $1 \mathrm{~A}$ ). Since there is no need of a dedicated perfusion specialist, the logistics becomes much easier. On the other hand, central implantation via thoracotomy which was performed in this case, as well as peripheral surgical implantation by cut down/vessel exposure, is being nowadays replaced by percutaneous femoral implantation $[5,6]$. Because front wall femoral puncture and fluoroscopy-guided cannula placement using supportive extra stiff wires are the key safety measures, the catheterization laboratory with a trained interventional cardiologist is considered to be

\section{Corresponding author:}

Marko Noc MD, PhD, Center for Intensive Internal Medicine, University Medical Center Ljubljana, Zaloska 7, 1000 Ljubljana, Slovenia, phone: +38615222296, e-mail: marko.noc@mf.uni-lj.si

Received: 24.09.2015, accepted: 24.09.2015. 

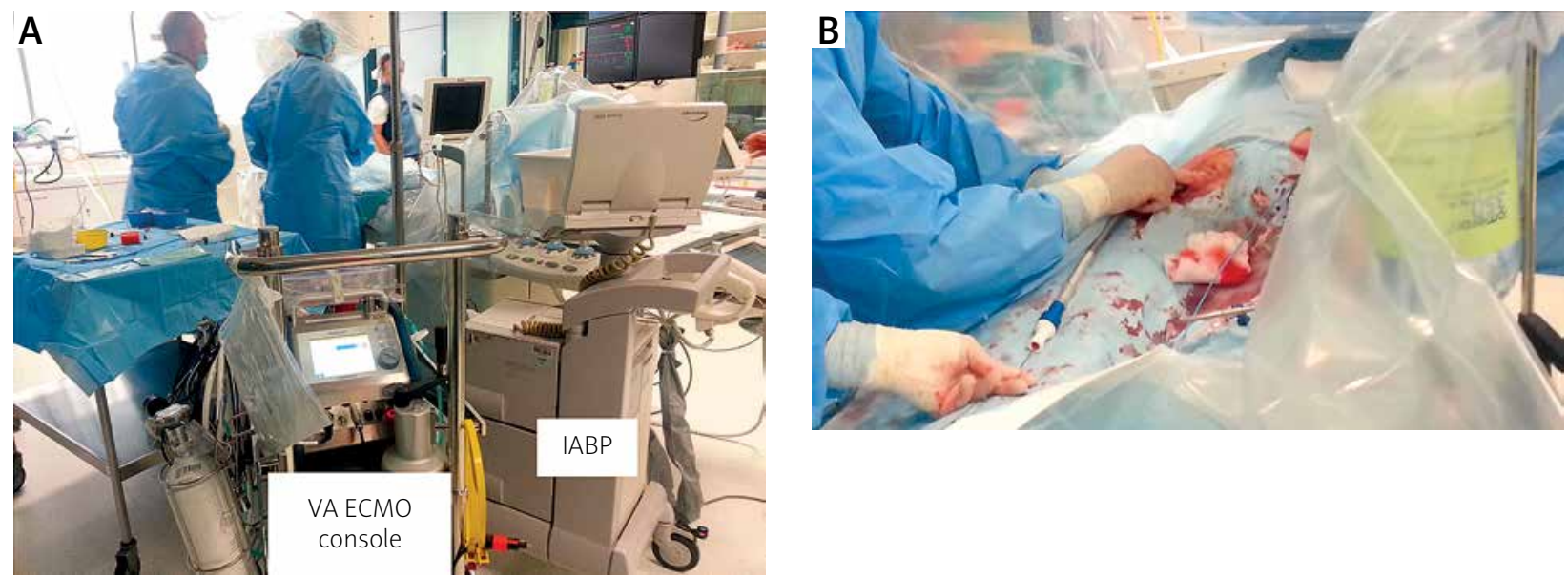

Figure 1. VA ECMO console ready to be used next to IABP in the catheterization laboratory (A). Fluoroscopy-guided percutaneous implantation of venous VA ECMO cannula in the right groin (B)

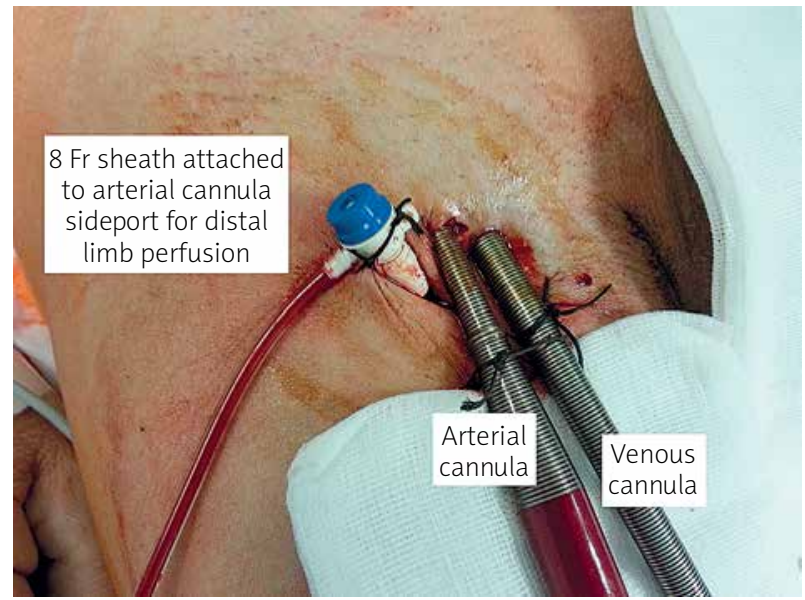

Figure 2. Right groin after percutaneous implantation of VA ECMO. Arterial and venous cannula are shown together with anterograde sheath for ipsilateral limb perfusion attached to arterial cannula via the side port

an excellent environment for percutaneous implantation [7] (Figure 1 B). Moreover, infrarenal angiography, which can be readily performed in the catheterization laboratory, facilitates selection of the least diseased site for arterial cannula and upfront implantation of the distal perfusion sheath to prevent ipsilateral limb ischemia [5, 8] (Figure 2). Implantation of the VA ECMO device in the catheterization laboratory is therefore rather easy and quick for a skilled interventional cardiologist, and there is no need for excessive training. I believe that a much more critical link in the management of VA ECMO patients is the acute cardiac care intensivist, who should join the interventional cardiologist already in the catheterization laboratory and take over ECMO setup, monitoring and intensive care treatment. A skilled intensive care unit team with 24-7 immediate access to cardiovascular surgery, an advanced heart failure program and other medical sub- specialties are crucial for ultimate survival of these patients. Since all these facilities are not available in every 24-7 cardiac interventional center, these patients should be centralized to a tertiary institution once stabilized on ECMO. There is no doubt that such transportation of patients on VA ECMO either by ambulance or helicopter can be safely performed.

In conclusion, I expect that the place of IABP in the catheterization laboratory, which was our good neighbor for many years, will soon be taken by one of the younger and more powerful cousins. Whether percutaneous VA ECMO is the best alternative remains as yet unknown. Importantly, the answer may differ from institution to institution, which may also choose between newcomers such as the more powerful percutaneous Impella CP and Thoratec $\mathrm{PHP}$, probably soon available also for routine clinical use.

\section{Conflict of interest}

Marko Noc received a speaker honorarium from Maquet Gettinge Group (Rastatt, Germany).

\section{References}

1. Pyka L, Pres D, Przybylski R, et al. Mechanical circulatory support in cardiogenic shock - what every interventional cardiologist should know. Postep Kardiol Inter 2014; 10: 195-200.

2. Litwiński P, Dębski A, Tyczyński P, et al. Rescue extracorporeal membrane oxygenation for refractory cardiogenic shock. Postep Kardiol Inter 2015; 11: 327-9.

3. Knafelj R, Trunk P, Terseglav S, et al. Rescue venoarterial ECMO in cardiogenic shock complicated by refractory cardiac arrest during percutaneous coronary intervention. Cor et Vasa 2014; 56: e348-353.

4. Ksela J, Knafelj R, Sostaric M, Noc M. Rescue veno-arterial extracorporeal membrane oxygenation and embolectomy for massive pulmonary thromboembolism with refractory cardiac arrest. Kardiol Pol 2015; in press.

5. Lamb KM, Hirose H, Cavarocchi NC. Preparation and technical considerations for percutaneous cannulation for veno-arterial extracorporeal membrane oxygenation. J Card Surg 2013; 28: 190-2. 
6. Benassi F, Vezzani A, Vignali L, Gherli T. Ultrasound guided femoral cannulation and percutaneous perfusion of the distal limb for VA ECMO. J Card Surg 2014; 29: 427-9.

7. Artl M, Phillipp A, Voelkel S, et al. Early experience with miniaturized extracorporeal life-support in the catheterization laboratory. Eur J Cardiothoracic Surg 2012; 42: 858-63.

8. Vander Salm TJ. Prevention of lower extremity ischemia during cardiopulmonary bypass via femoral cannulation. An Thorac Surg 1997; 63: 251-2. 